\title{
Do Scores on an Attention Test Predict Scores on Executive Function Tests?
}

\author{
Angela N. Burda, Jaimie L. Gilbert, Courtney Amundson, Kelsey Baughman, Aaron Brummel, Sarah Crimmins, \\ Lisa Daringer, Courtney Hansen, Delaney Hoffman, Olivia Ferguson, Katherine Polit
}

Department of Communication Sciences and Disorders, University of Northern lowa, Cedar Falls, IA, USA

Purpose: This study sought to determine if younger and older adults' scores on the Attention Process Test predicted scores on the Behavioral Assessment of Dysexecutive Syndrome and Functional Assessment of Verbal Reasoning and Executive Strategies.

Methods: The Attention Process Test, the Behavioral Assessment of Dysexecutive Syndrome, and the Functional Assessment of Verbal Reasoning and Executive Strategies were administered to 60 younger and 60 older adults in a counter-balanced manner. Regression statistics were calculated to determine any predictive outcomes.

Results: For older adults, the Attention Process Test significantly predicted the Behavioral Assessment of Dysexecutive Syndrome's total profile standard scores, and the Functional Assessment of Verbal Reasoning and Executive Strategies' total standard scores for Accuracy, Rationale, and in one analysis significantly predicted Reasoning. For younger adults, when correcting for high correlations among the five Attention Process Test subtests, this test did not significantly predict their scores on the Behavioral Assessment of Dysexecutive Syndrome or the Functional Assessment of Verbal Reasoning and Executive Strategies' total standard scores.

Conclusions: The Attention Process Test was consistently a significant predictor of executive function measures in older adults, but not younger adults, suggesting the relationship between attention and executive function may be different for these age groups. Thus, different evaluation procedures may be warranted for older adults versus younger adults. A caveat of these findings is that these results were obtained with healthy, neurologically intact individuals. Future research should further investigate the relationship between attention and executive function in adults with acquired neurological damage.

Keywords: Adult, Aging, Attention, Cognition, Executive function

\section{INTRODUCTION}

Cognition encompasses many different abilities, including attention and executive functions. Yogev-Seligmann et al. [1] define attention as the ability to receive a stimulus and correctly respond to the stimulation. In essence, individuals orient and alert to stimuli $[2,3]$ and decide if the focus should continue to be processed or ignored [4]. Several different types of attention exist including selective, divided, sustained, and alternating attention $[2,5]$. Selective attention is described as the ability to concentrate on a certain stimulus (e.g., auditory, visual) and suppress irrelevant factors [6,7]. Gaspelin

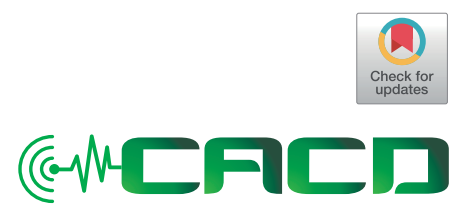

Received: September 7, 2018 Revision: December 18, 2018 Accepted: December 25, 2018

Correspondence:

Angela N. Burda

Department of Communication Sciences and Disorders, University of Northern lowa, 230 Communication Arts Center Cedar Falls, IA 50614-0356, USA

Tel: $+319-273-3128$

Fax: +319-273-6384

E-mail: angela.burda@uni.edu

C) 2018 The Korean Association of SpeechLanguage Pathologists

This is an Open Access article distributed under the terms of the Creative Commons Attribution NonCommercial License (http://creativecommons.org/ licenses/by-nc/4.0/) which permits unrestricted noncommercial use, distribution, and reproduction in any medium, provided the original work is properly cited. 
et al. [8] report selective attention can be both voluntary and involuntary in nature and describe it as focusing on one part at a time (e.g., different objects, tasks, locations). Divided attention, on the other hand, is parallel to multi-tasking and involves spreading attentional resources to different targets (e.g., speakers, spatial locations) $[1,6]$. Sustained attention is the ability to stay focused on and respond to a specific stimulus for a certain period of time [1,9-11]. Alternating, or switching, attention is the act of rapidly and continuously transferring from one task, ability, or cognitive set to another $[1,9,11,12]$.

Researchers have investigated if attentional abilities change with age. In general, older adults have more difficulty with more complex tasks compared to younger adults [13-15]. Research has also been conducted on the more specific types of attention. For example, alternating attention may decline during aging when switching between cognitive sets, however, this decline may be lessened with cognitive training [12]. Regarding divided attention, this type of attention may be more greatly affected by age than other types of attention [1], and younger adults can have higher performance on divided attention tasks compared to older adults [16]. For selective attention, although Aydin, Strang, and Manahilov (2013) suggested it tends to remain consistent between older and younger adults, Gaspelin, Ruthruff, and Lien (2016) found older adults may display greater difficulty than younger adults if presented with more challenging tasks $[8,12]$. Somewhat contradictory results have been reported on sustained attention tasks, however, in general, it appears to remain fairly consistent in younger and older adults [12,17]. Thus, older adults' performance on selective and sustained attention tasks appears to be consistent with younger adults except when tasks are more challenging. Alternating attention, and especially divided attention, appear to be the most challenging for older adults and can evidence possible age-related declines.

Executive function (EF) is another broad area of cognition. Although multiple definitions exist regarding EF, most tend to refer to a variety of higher cognitive processes that use and modify information and can include: sequencing and organizing information, demonstrating self-control, shifting (task switching), updating (monitoring and manipulating relevant information in working memory), choosing, and if necessary, inhibiting a response $[1,18,19]$. The presence of adequate $\mathrm{EF}$ is essential in helping individuals determine a situation, establish a goal, create a plan to accomplish the desired goal, and put that plan into action, altering it as needed, while selfmonitoring throughout the entire process [18]. EF also has important pragmatic implications by allowing individuals to have appropriate discourse skills and the ability to adapt and adjust their behaviors to new situations or prevent inappropriate behaviors from occurring [20].

As with attentional abilities, researchers have attempted to determine if $\mathrm{EF}$ changes with age. Young adults appear to perform better on tests assessing EF compared to older adults [21-23]. Burda et al. (2017) administered the Behavioural Assessment of Dysexecutive Syndrome [24] and the Functional Assessment of Verbal Reasoning and Executive Strategies [25] to 105 younger (age 20-39 years), middle-aged (age 40-59 years) and older (age 60 or older) participants. Younger adults had significantly higher scores on both EF tests compared to the older adults, even though all participants were self-reported to be healthy and lived independently. Yet, YogevSeligmann et al. [1] suggested that EF can remain normal throughout the aging process and that multiple factors such as declines in mental flexibility and abstract thinking may contribute to a reduction of EF performance, not just aging alone. Also, Dahlin et al. [26] found that a targeted intervention improved EF performance in older adults, although more significantly so in younger adults. Thus, while older adults can exhibit declines in EF, not all researchers agree on the underlying reason, and EF can potentially improve through targeted training. In addition, attention and EF skills may be interconnected. For example, it is plausible that controlled attention could be the basis for EF [19].

Speech-language pathologists (SLPs) can use various tests to measure attention and EF skills in adults, including the Attention Process Training Test [10] to measure the various types of attention, and the BADS and the FAVRES to evaluate $\mathrm{EF}[24,25]$. The APT Test is a screening tool that assesses simple sustained, complex sustained, selective, divided, and alternating attention. The examiner reads aloud the instructions to participants and then plays an audio $\mathrm{CD}$ for each subtest (i.e., level) in which a narrator reads a set of numbers. Participants utilize a clicker to indicate the correct stimulus (e.g., click when they hear the number "two" being said during the simple sustained attention subtest). There are a total of five levels with each subtest increasing in difficulty. For example, as noted, participants click when they hear the number "two" in the simple sustained attention subtest (level 1). During the complex sustained subtest (level 2), participants press the clicker when they hear a number that is one less than the number immediately before it. For the selective attention subtest (level 3), individuals click when they hear the 
number "two" while distracting background information is simultaneously being played. During the divided attention task (level 4), individuals are required to click when they hear the number "two" being played while also using a writing utensil to cross off even numbers in a large set of numbers on a sheet of paper. Finally, for the alternating attention task (level 5), participants press the clicker when they hear a number that is one less than the number immediately before it. The examiner then says "switch" during the audio presentation, and participants must press the clicker when they hear a number that is one more than the number immediately before it. These switching events occur several times throughout the subtest. The test can be administered generally within 20-30 minutes. While the APT Test was originally normed on a sample of 25 control individuals $\left(\mathrm{M}_{\text {age }}=26.3\right)$ and 25 individuals with mild brain injuries $\left(\mathrm{M}_{\mathrm{age}}=27.2\right)$, the examiner's manual reports means and standard deviations for the following age groups: $18-29$ years, $30-39$ years, $40-59$ years, and $60-85$ years old [1].

Burda et al. (2017) and Kallambettu, Burda, and Wakeman (2017) recently described the BADS and FAVRES [21,24,25,27]. The BADS has six tests (i.e., subtests) measuring various EF abilities (e.g., organizing, adapting behavior) and requires verbal, written, and hands-on responses. The first test has participants learn a rule pertaining to a series of cards, carry out the specified task, unlearn the first rule, rapidly learn a different rule, and carry out the newly specified task. Additional tests include, but are not limited to, individuals: answering temporal judgement questions, mapping out routes to visit different exhibits at a zoo following certain rules, and drawing a search pattern when trying to find lost keys in a field. The BADS takes approximately 40-50 minutes to administer and was normed on 78 persons aged 19-78 years with differing neurological disorders and 216 healthy adults divided into four age groups: 16-31, 32-47, 48-63, and 64 years and older [24].

Persons complete four ecologically valid subtests on the FAVRES, such as scheduling one's workday or writing a letter of complaint for poor workmanship on a home repair. Participants provide verbal and written responses to each of the four situations, all of which include various restrictions (e.g., repair company lost the original contract). Individuals must also complete prediction and generation tasks for each subtest. For the poor home repair subtest, participants generate a list of everything that might be of value in their own home that would cost money to replace. They then predict two problems that could occur from hiring a new contractor and billing the original contractor for the new repair. Administration time is generally 50-60 minutes (MacDonald \& Johnson, 2005). The FAVRES was normed on 52 adults with acquired brain injury and 101 healthy adults aged 17-89 years [25].

\section{Purpose and research question}

Despite the research that has been conducted in the areas of attention and $\mathrm{EF}$, little research has been conducted to determine possible effects attentional abilities can have on EF outcomes. Yet, these broad cognitive abilities are essential in order for individuals to be successful and independent in their daily lives [20]. If scores on attentional abilities could predict scores on EF tests, it could lead to a potentially more efficient evaluation process as well as provide a better understanding of the relation between these various skill sets. Few studies have been found which include the tests described above (i.e., APT Test, BADS, FAVRES), however, these tests may be used by practicing SLPs who are continually tasked with assessing their patients' cognitive abilities [28]. Knowing if scores on an attention test could predict executive function test scores could add to the present literature base and ideally help ensure SLPs are adequately evaluating and ultimately treating adult patients with cognitive-communicative impairments. This study aims to address the following research question: Does the APT Test predicts scores on the BADS and FAVRES in younger and older adults?

\section{METHODS}

\section{Participants}

Participants for this cross-sectional quasi-experimental study were recruited from small, mid-sized, and large urban and rural Midwestern communities. A power analysis for an effect size of 0.08 with an alpha of 0.05 indicated a minimum total sample size of 70 was needed, however, a total of 120 adults participated and were equally divided into two groups. The 60 younger adults ranged in age from 18-40 years $\left(\mathrm{M}_{\text {age }}=23.77\right.$ years; $S D=3.86$ ); the 60 older adults were aged 60 years and older $\left(\mathrm{M}_{\text {age }}=68.80 ; S D=7.64\right)$. The study was approved by the University of Northern Iowa's Institutional Review Board (Protocol \# 14-0241); all ethical guidelines were adhered to, and all individuals provided informed consent to participate. Similar to Burda et al. [21], inclusion criteria included: no history of neurological impairment, events, or disorders; be a native English speaker; have a minimum of a high school level of education; and score a 28 or higher on the Mini-Mental State Ex- 
amination [29]. Young adults had a $\mathrm{M}_{\text {score }}=29.80(S D=0.61)$ on the MMSE, while older adults had a $\mathrm{M}_{\text {score }}=29.15$ ( $S D=$ 0.95). Hearing screenings were not conducted. Researchers in a prior study using the APT Test with older adults did not screen participants' hearing [30]. In the current study, all directions and items were presented at a volume level that was comfortable for each participant and adjusted as needed.

\section{Stimuli, procedures, and data analysis}

Participants were individually administered the APT Test, BADS and FAVRES in a counterbalanced order and according to the respective examiners' manuals. Screening and testing measures were conducted in a single session lasting approximately 120 minutes; breaks were allowed as needed.

Participant responses were scored according to the procedures found in each of the test manuals. For the APT Test, residual (i.e., total) scores (TH-FP) for each subtest were determined by subtracting the number of false positives (FP) from the number of correct target hits (TH) [10]. Target hits refer to the number of times a participant pressed the clicker in response to correct target. False positives refer to the number of times the clicker was pressed when it should not have been. Except for alternating attention, in which the possible maximum score was 24 , the total score for the remaining four subtests of the APT Test was 30.

Data analysis of the BADS and the FAVRES followed the format used in previous studies [21,27,31]. Raw scores on the BADS were converted to Profile Scores that ranged from 0 to 4 . Certain subtests had timed components that were used to calculate the Profile Score. An overall Profile Score was calculated by summing all Profile Scores for each of the six tests. Participants earned a Total Profile Score ranging from 0 to 24 if the entire test was completed in its entirety. For the FAVRES, participants earned four raw scores for each Task (i.e., subtest): Accuracy, Rationale, Time, and Reasoning Subskills. Individuals received the highest possible points for the most ap- propriate answer; some, but not all, points were given for reasonable related responses. Raw scores were converted into standard scores $(M=100, S D=15)$ for Accuracy, Rationale, Time, and Reasoning and also used to calculate using the test's Total Score.

\section{Reliability}

Pearson $r$ correlations were calculated using subtest raw scores on $20 \%$ of a randomly chosen sample (i.e., 24 participants). The investigators and a trained speech-language pathology student's scores were correlated for inter-rater reliability. Investigators scored the protocols twice for intra-rater reliability; the second scoring took place two weeks following the initial scoring. Inter-rater reliability for the APT Test was $r=0.95$; intra-rater reliability was $r=0.97$. Inter-rater reliability for the BADS was $r=0.91$; intra-rater reliability was $r=0.92$. Inter-rater reliability for the FAVRES was $r=0.89$; intra-rater reliability was $r=0.93$

\section{RESULTS}

\section{APT Test}

Means and standard deviations of residuals for the five subtests are presented in Table 1. Older adults' average performance fell within or above reported norms (+/- 1 standard deviation) for 60-85 year olds [10]. Younger adults' average performance fell within reported norms (+/- 1 standard deviation) for 18-29 year olds [10].

\section{BADS}

Standard scores (i.e., mean of 100, standard deviation of 15), based on the Total Profile Score across all six subtests were calculated according to normed controls reported in the manual [24]. Older adults averaged a standard score of 94.97 $(S D=14.13)$ on the BADS. Younger adults averaged a standard score of $102.83(S D=9.97)$ on the BADS. Table 2 includes both age groups' mean scores and standard deviations for this test.

Table 1. Mean and standard deviations of APT Test subtest scores for younger and older adults

\begin{tabular}{|c|c|c|c|c|c|}
\hline \multirow{2}{*}{ APT Test subtests } & \multirow{2}{*}{$\begin{array}{c}\text { Total score } \\
\text { possible }\end{array}$} & \multicolumn{2}{|c|}{ Younger adults $(n=60)$} & \multicolumn{2}{|c|}{ Older adults $(n=60)$} \\
\hline & & $M$ & $S D$ & $M$ & $S D$ \\
\hline Sustained attention & 30 & 29.78 & 1.30 & 29.87 & 0.57 \\
\hline Complex sustained attention & 30 & 25.82 & 4.20 & 20.65 & 6.61 \\
\hline Selective attention & 30 & 26.55 & 3.45 & 21.70 & 7.20 \\
\hline Divided attention & 30 & 28.47 & 1.42 & 25.97 & 3.47 \\
\hline Alternating attention & 24 & 20.35 & 3.75 & 15.98 & 5.34 \\
\hline
\end{tabular}


Table 2. Mean and standard deviations of bads profile scores for younger and older adults

\begin{tabular}{|c|c|c|c|c|c|}
\hline \multirow[t]{2}{*}{ BADS subtests } & \multirow{2}{*}{$\begin{array}{c}\text { Total } \\
\text { score } \\
\text { possible }\end{array}$} & \multicolumn{2}{|c|}{$\begin{array}{l}\text { Younger adults } \\
\qquad(n=60)\end{array}$} & \multicolumn{2}{|c|}{$\begin{array}{l}\text { Older adults } \\
\qquad(n=60)\end{array}$} \\
\hline & & $M$ & $S D$ & $M$ & $S D$ \\
\hline Rule-shift cards & 4 & 3.70 & 0.46 & 3.67 & 0.84 \\
\hline Action program & 4 & 3.82 & 0.79 & 3.67 & 0.84 \\
\hline Key search & 4 & 3.10 & 0.99 & 2.75 & 1.23 \\
\hline $\begin{array}{l}\text { Temporal } \\
\text { judgment }\end{array}$ & 4 & 1.20 & 0.66 & 1.22 & 0.61 \\
\hline Zoo map & 4 & 3.12 & 0.83 & 2.52 & 1.24 \\
\hline $\begin{array}{l}\text { Modified six } \\
\text { elements }\end{array}$ & 4 & 3.77 & 0.50 & 3.48 & 0.87 \\
\hline $\begin{array}{l}\text { Total points } \\
\text { score }\end{array}$ & 24 & 18.68 & 2.08 & 17.05 & 2.90 \\
\hline Standard score ${ }^{*}$ & 100 & 102.83 & 9.97 & 94.97 & 14.13 \\
\hline
\end{tabular}

*Denotes mean standard score and not the total score possible.

\section{FAVRES}

Total scores were calculated for Accuracy, Rationale, Time, and Reasoning. The total scores for each type of analysis (e.g. Accuracy) were then converted into standard scores, based on normative tables reported in the manual [25]. Mean standard scores for both age groups are presented in Table 3.

\section{Regression analyses}

Multiple regression analyses were performed to assess if measures of attention predicted performance on executive function tests for older and for younger adults. Models were evaluated for overall significance, and only significant coefficients are reported in the regression equations. For all tests of significance, alpha level was set at $p<0.01$. A correlation analysis of the combined age groups $(n=120)$ revealed that all APT Test subtest residual scores were significantly correlated with each other, with the exception of Sustained attention not correlated with any of the other subtests. Due to multi-collinearity among the APT Test subtests, a transformed score of the sum of all five APT Test residual scores was created, and simple linear regression analyses were also completed.

\section{Does APT Test predict BADS performance?}

A multiple regression analysis was performed to evaluate if attention, as assessed by the APT Test, could predict executive function abilities, as assessed by the BADS Total Profile Score. Independent variables consisted of the five residual scores for each of the APT Test subtests, with the dependent variable of
Table 3. Means and Standard Deviations of FAVRES Standard Scores for Younger and Older Adults

\begin{tabular}{|c|c|c|c|c|c|}
\hline \multirow[t]{2}{*}{ FAVRES tasks } & \multirow{2}{*}{$\begin{array}{l}\text { Total SS } \\
\text { possible }\end{array}$} & \multicolumn{2}{|c|}{$\begin{array}{l}\text { Young adults } \\
\qquad(n=60)\end{array}$} & \multicolumn{2}{|c|}{$\begin{array}{l}\text { Older adults } \\
\qquad(n=60)\end{array}$} \\
\hline & & $M$ & $S D$ & $M$ & $S D$ \\
\hline \multicolumn{6}{|l|}{ Accuracy SS } \\
\hline Task 1 & 108 & 103.25 & 10.85 & 90.58 & 19.25 \\
\hline Task 2 & 106 & 91.53 & 19.74 & 81.93 & 29.66 \\
\hline Task 3 & 107 & 93.47 & 19.88 & 93.42 & 19.54 \\
\hline Task 4 & 106 & 82.77 & 27.78 & 69.35 & 34.01 \\
\hline Total Test & 111 & 90.37 & 16.58 & 75.55 & 27.73 \\
\hline \multicolumn{6}{|l|}{ Rationale SS } \\
\hline Task 1 & 106 & 103.53 & 9.31 & 93.25 & 28.89 \\
\hline Task 2 & 109 & 99.48 & 13.90 & 87.93 & 17.83 \\
\hline Task 3 & 103 & 94.80 & 17.62 & 92.87 & 26.79 \\
\hline Task 4 & 107 & 67.17 & 35.01 & 66.87 & 33.19 \\
\hline Total Test & 111 & 84.58 & 20.56 & 74.32 & 25.58 \\
\hline \multicolumn{6}{|l|}{ Time SS } \\
\hline Task 1 & 132 & 111.47 & 11.43 & 107.45 & 17.66 \\
\hline Task 2 & 144 & 101.68 & 23.46 & 92.13 & 26.68 \\
\hline Task 3 & 130 & 108.38 & 9.71 & 102.97 & 14.34 \\
\hline Task 4 & 135 & 111.08 & 9.10 & 106.43 & 16.99 \\
\hline Total Test & 126 & 110.62 & 12.87 & 100.85 & 19.08 \\
\hline Reasoning SS & 142 & 98.40 & 15.35 & 71.17 & 32.40 \\
\hline
\end{tabular}

SS, standard score.

the BADS Total Profile Score. Analyses were performed separately for the older adults and younger adults. The model was significant for older adults $[F(5,54)=7.90 p<0.001]$, accounting for approximately $42 \%$ of the variance in BADS Total Profile Scores $\left(R^{2}=0.42\right)$. The BADS Total Profile Score was equal to $48.74+1.73$ (Alternating Residual); none of the other APT Test subtest coefficients were significant. The model was also significant for younger adults $[F(5,54)=4.90, p=0.001]$, accounting for approximately $31 \%$ of the variance in BADS Total Profile Scores $\left(R^{2}=0.31\right)$. For younger adults, the BADS Total Profile Score was equal to $17.93+2.72$ (Divided Residual)-1.42 (Selective Residual)+1.29 (Complex Sustained Residual). A simple linear regression with the APT Test subtest scores summed together to create one score was performed. This model was only significant for older adults $[F(1,58)=24.22, p<0.001$; $\left.R^{2}=0.30\right]$.

\section{Does APT Test predict FAVRES performance?}

Multiple regression analyses were performed to determine 
prediction strength of the APT Test on the four different measures of the FAVRES: Accuracy, Rationale, Time, and Reasoning. The five subtests of the APT Test were again the independent variables, with each of the FAVRES measures total standard score being the dependent variable in separate regression models. Again due to multi-collinearity among the APT Test subtests, linear regression results with one transformed (summed) APT Test score as the independent variable are also reported.

\section{Accuracy}

The five subtests of the APT Test significantly predicted FAVRES Accuracy for older adults $[F(5,54)=7.79, p<0.001]$. The regression model accounted for approximately $42 \%$ of the variance in FAVRES accuracy $\left(R^{2}=0.42\right)$ with FAVRES Accuracy standard score $=-294.54+2.36$ (Alternating Residual). The transformed, single independent variable of the sum of APT Test subtest residual scores was again only a significant predictor of FAVRES Accuracy for older adults $[F(1,58)=28.14$, $p<0.001 ; R^{2}=0.33$.

\section{Rationale}

APT Test performance on the five subtests significantly predicted FAVRES Rationale total standard score only for older adults $[F(5,54)=5.47, p<0.001]$. The complete model accounted for approximately $34 \%$ of the variance in Rationale measures $\left(R^{2}=0.34\right)$, with no individual subtest coefficient reaching significance. Linear regression analysis with the transformed summed APT Test score was also only significant for older adults $\left[F(1,58)=16.27, p<0.001 ; R^{2}=0.22\right]$.

\section{Time and reasoning}

APT Test scores did not significantly predict FAVRES Time total standard scores for any analysis, for either age group. Only the simple linear regression using the summed residual APT Test score significantly predicted FAVRES Reasoning standard scores, and only for older adults $[F(1,58)=13.98, p<0.001$; $\left.R^{2}=0.19\right]$.

\section{DISCUSSION AND CONCLUSIONS}

Executive function is complex and multi-faceted and, therefore, can be evaluated with different assessments. Two such assessments were included in the current study: the BADS and FAVRES. Regression analyses were performed to determine if attention skills, as measured by the APT Test, pre- dicted EF ability for a group of older adults and a group of younger adults. For older adults, the APT Test significantly predicted the BADS total profile standard scores, accounting for $30-42 \%$ of the variance. For older adults, the APT Test also significantly predicted the FAVRES total standard scores for Accuracy (33-42\% of the variance), Rationale (22-34\% of the variance), and in one analysis significantly predicted Reasoning (19\% of the variance). For younger adults, in one analysis, the APT Test significantly predicted the BADS profile score accounting for $31 \%$ of variance. However, when correcting for high correlations among the five APT Test subtests, the APT Test did not significantly predict BADS scores for younger adults. The APT Test also did not significantly predict any FAVRES total standard scores for young adults.

The purpose of this study was to determine if the APT Test Test predicts scores on the BADS and FAVRES in younger and older adults. The results suggest that the relationship between attention and executive function may be different for older adults compared to younger adults. That is, the APT Test was consistently a significant predictor of EF measures for older adults, but not for younger adults. Previous research has suggested that older adults' poorer executive function may be related to the use of less efficient strategies [32]. It is possible that the strategies employed by older adults, but not younger adults, to perform executive function tasks may utilize similar resources as attention, contributing to the current findings.

\section{Clinical implications}

SLPs assess cognitive skills as an essential component when developing cognitive-communicative treatment goals for a variety of individuals, including those who may have aphasia, been diagnosed with a neuro-degenerative disease, or experienced traumatic brain injury [33]. The current study suggests that different evaluation procedures may be warranted for older adults than for younger adults due to age differences in the relationship between attention and executive function. A caveat of these findings is that these results were obtained with healthy, neurologically intact individuals. Questions remain as to the relationship between attention and executive function in the presence of neurological insult.

On a practical note, the APT Test which assesses attention, takes approximately 20 minutes to complete compared to a longer administration time for the executive function assessments used in this study, which ranged from approximately 40-60 minutes per test. In a busy medical environment, a possible approach for assessment of older adults may be to assess 
attention and determine if a follow-up assessment of executive function is warranted (i.e., if attentional skills are poor). It should be noted that although attention was a consistent significant predictor of executive function, it by no means explained all the variance in executive function scores. Therefore, an evaluation of attention may be a good screening measure for older adults, but the complete assessment battery, as always, would need to be determined on a case by case basis. However, for younger adults, since attention was not found to consistently predict executive function test scores, evidence from this study suggests that both attention and executive function would need to be assessed.

In addition to implications for assessment procedures, there is a need to further explore differences in treatment efficacy for individuals of different ages. For example, training on executive function tasks has been shown to have varying outcomes for older and younger adults [34]. The relationship between attention and executive function in older adults warrants more attention to develop age appropriate, beneficial, efficient, and efficacious therapeutic interventions.

\section{Limitations and future research}

Several limitations exist within this study, including a lack of randomization of participants which resulted in participant groups who were generally younger in nature in both age ranges (i.e., younger adults $\mathrm{M}_{\mathrm{age}}=23.8$ years; older adults, $\mathrm{M}_{\mathrm{age}}=68.8$ years), highly educated, and did not represent culturally and linguistically diverse (CLD) populations. While it may not be likely that persons in their 30s would perform significantly differently than those in their 20s, it is certainly plausible individuals in their 80s and 90s may perform differently than those in their 60s. Adults older than age 75 (i.e., oldold) have demonstrated poorer skills than older adults aged less than 75 years (i.e., young-old) in various cognitive abilities and tasks, including perceptual speed and episodic memory [35,36], digit retention [37], verbal fluency [38] , and working memory [39]. Conversely, Calero \& Navarro [36] analyzed the effects of aging and cognitive status (healthy vs. cognitively impaired) between young-old adults and old-old adults. Other than long-term memory measures indicating age-related differences, healthy older adults performed significantly better than those who were cognitively impaired on other cognitive measures [36]. Thus, cognitive status, not age alone, may determine how well older adults perform on the various cognitive tasks. Nonetheless, older adult participants were relatively young in the current study.
Participants were also highly educated and few represented CLD populations. Only eight younger adults and 27 older adults had a 2-year degree or less in terms of their level of education. Individuals with higher education levels can have higher scores on tasks measuring attention and EF $[40,41]$. Therefore, it is difficult to say if participants who were not as highly educated would have had similar outcomes. Also, recent studies have reported that adults representing CLD populations have had differing performance on the BADS and FAVRES compared to Caucasian adults $[27,42]$. It is unknown if the current study's results would have differed had there been a greater number of persons representing CLD populations. Finally, participants were not asked to report any over-thecounter or prescribed medications they were taking. However, various medications, such as those used to treat seizure disorders (e.g., topiramate and tiagabine), vertigo (e.g., diphenhydramine hydrochloride), and depressive disorders with an anxiety component (e.g., trazodone), can lead to declines in cognitive abilities (e.g., working memory, verbal memory) [43-45].

Since SLPs working in medical settings could possibly use the tests included in the current study with their adult patients, any potential findings of future studies would ideally inform clinical practice. Thus, forthcoming research could include replicating the study with randomized participants that better represent various ages, education levels, and CLD populations. This study could also be replicated with younger and older adults who have acquired neurological damage and possibly additional diagnoses. For example, persons who have certain comorbidities (e.g., cardiovascular infarcts, diabetes) can be at an increased chance of developing cognitive deficits [46]. Latency measurements of responses between younger and older adults could be measured to determine potential differences between the studied groups. The researchers informally observed that most of the older adults took longer to press the clicker on the APT Test versus younger adults. Older adults often demonstrate slower reaction times [47] and reaction times can be a possible indicator of cognitive function [48]. Investigating whether or not latency response on the APT Test has an effect on or predicts performance on the BADS and FAVRES in differently-aged adults could add to the literature base. Finally, future research could determine if treating attention in older adults leads to changes in their executive function test performance. 


\section{ACKNOWLEDGMENTS}

Funding was provided by the University of Northern Iowa's College of Humanities, Arts, and Sciences Small Grant and the Graduate Research Award for Student Projects.

\section{CONFERENCE PRESENTATION}

This study was presented November 2017 at the Annual Meeting of the American Speech-Language-Hearing Association in Los Angeles, California.

\section{REFERENCES}

1. Yogev-Seligmann G, Hausdorff JM, Giladi N. The role of executive function and attention in gait. Mov Disord. 2008;23:329-342.

2. Peterson DS, King LA, Cohen RG, Horak FB. Cognitive contributions to freezing of gait in Parkinson disease: Implications for physical rehabilitation. Phys Ther. 2016;96:659-670.

3. Posner M, Petersen S. The attention system of the human brain. Annu Rev Neurosci. 1990;13:25-42.

4. Marshall SD. Helping experts and expert teams perform under duress: an agenda for cognitive aid research. Anaesthesia. 2017; 72:289-295.

5. Mcdowd JM. An overview of attention: behavior and brain. J Neurol Phys Ther. 2007;31:98-103.

6. Getzmann S, Golob EJ, Wascher E. Focused and divided attention in a stimulated cocktail-party situation: ERP evidence from younger and older adults. Neurobiol Aging. 2016;41:138-149.

7. Kueider AM, Parisi JM, Gross AL, Rebok GW. Computerized cognitive training for older adults: a systematic review. PloS One. 2012 [cited 2018 Jun 5]; 7.

8. Gaspelin N, Ruthruff E, Mei-Ching L. The problem of latent attentional capture: easy visual search conceal scapture by task-irrelevant abrupt onsets. J Exp Psychol Hum Percept Perform. 2016;42: 1104-1120.

9. Lezak MD, Howieson DB, Bigler ED, Tranel D. Neuropsychological assessment. 5th ed. New York: Oxford University press; 2012.

10. Sohlberg MM, Mateer C. APT-3: Attention Process Training. North Carolina: Lash \& Associates; 2001.

11. Rogers WA. Attention and aging. In: Park DC, Schwarz N, editors. Cognitive aging: a primer. Pennsylvania: Psychology Press; 2006. p. 57-71.

12. Zanto TP, Gazzaley A. Attention and aging. In: Nobre K, Kastner S, editors. The oxford book of attention. United Kingdom: Oxford University press; 2014. p. 927-941.

13. Carlson MC, Hasher L, Connelly SL, Zacks RT. Aging, distraction, and the benefits of predictable location. Psych and Aging. 1995; 10:427.

14. Harada CN, Love MCN, Triebel KL. Normal cognitive aging. Clin Geriatr Med. 2013;29.
15. Salthouse TA, Fristoe NM, Lineweaver TT, Coon VE. Aging of attention: does the ability to divide decline? Mem Cogn. 1995;23:5971.

16. Anderson ND, Craik FI, Benjamin MN. The attentional demands of encoding and retrieval in younger and older adults: I. Edvidence from divided attention costs. Psychol Aging. 1998;13:405423.

17. Aydin S, Strang NC, Manhilov V. Age related deficits in attentional control of perceptual rivalry. Vision Res. 2013;77:32-40.

18. Keil K, Kaszniak AW. Examining executive function in individuals with brain injury: A review. Aphasiology 2002 March [cited 2018 Jun 15];16.

19. Miyake A, Friedman NP, Emerson MJ, Witzki AH, Howerter A, Wager TD. The unity and diversity of executive functions and their contributions to complex "frontal lobe" tasks: a latent variable analysis. Cognitive Psychology. 2000;41:49-100.

20. Jurado MB, Rosselli M. The elusive nature of executive functions: a review of our current understanding. Neuropsychol Rev. 2007;17: 213-233.

21. Burda A, Anderson E, Berryman M, Davis C, Heun M, Kise T. Performance of young, middle-aged and older adults on tests of executive function. CJSLPA. 2017;41:253-262.

22. Faria CA, Alves HVD, Charchat-Fichman H. The most frequently used tests for assessing executive functions in aging. Dement Neuropsychology. 2015;9:149-155.

23. Rouch I, Thomas Anterion C, Dauphinot V, Kerleroux J, Roche F, Barthelemy JC, et al. Cognitive complaints, neuropsychological performance and affective disorders in elderly community residents. Disability and Rehabilitation. 2008;30:1794-1802.

24. Wilson BA, Alderman N, Burgess PW, Emslie HC, Evans JJ. BADS: Behavioral Assessment of Dysexecutive Syndrome. United Kingdom: Thames Valley; 1996.

25. MacDonald S. FAVRES: Functional Assessment of Verbal Reasoning and Executive Strategies. Ontario: CCD Publishing; 2005.

26. Dahlin E, Nyberg L, Backmann L, Neely AS. Plasticity in executive functioning in younger and older adults: immediate training gains, transfer and long-term maintenance. Psychol Aging. 2008; 23:720-730.

27. Kallambettu V, Burda A, Wakeman N. South asian adults' performance on executive function tests. Am J Speech Lang Pathol. 2017;26:1254-1261.

28. Frith M, Togher L, Ferguson A, Levick W, Docking K. Assessment practices of speech-language pathologists for cognitive communication disorders following traumatic brain injury in adults: an international survey. Brain Inj. 2014;28:1657-1666.

29. Folstein M, Folstein S, McHugh P. MMSE: Mini Mental State Examination. Florida: Psychological Assessment Resources; 2001.

30. Sohlberg MM, McLaughlin KA, Pavese A, Heidrich A, Posner MI. Evaluation of attention process training and brain injury education in persons with acquired brain injury. J Clin Exp Neuropsychol. 2010;22:656-676.

31. Burda A, Baker K, Burns A, Butler C, Clark L, Cook W, et al. Performance of young and middle-aged adults on cognitive tests. J 
Med Speech Lang Pathol. 2014;21:393-404.

32. Hampshire A, Gruszka A, Fallon S, Owen A. Inefficiency in selforganized attentional switching in the normal aging population is associated with decreased activity in the ventrolateral prefrontal cortex. J Cogn Neurosci. 2008;20:1670-1686.

33. Sohlberg MM, Turkstra L. Optimizing cognitive rehabilitation: effective instructional methods. New York: The Gulford Press; 2011.

34. Dorbath L, Hasselhorn M, Titz C. Aging and executive function: a training study on focus-switching. Front Psychol. 2011;2:1-12.

35. Backman L, Small B, Larsson M. Cognitive Functioning very old age. In: Craik F, Salthouse T, editors. Handbook of cognitive aging. 2nd ed.. New Jersey: Lawrene Enbaum Associates; 200. p. 499-558.

36. Calero D, Navarro E. Differences in cognitive performance, level of dependency and quality of life (QoL), related to age and cognitive status in a sample of Spanish old adults under and over 80 years of age. Arch Gerentol Geriatr. 2011;53:292-297.

37. Read S, Volger GP, Pedersen NL, Johansson B. Stability and change in genetic and environmental components of personality in old age. Pers Individ Dif. 2006;40:1637-1647.

38. Snitz BE, Unverzagt FW, Chang CC, VanderBilt JG, Gao S, Saxton J, et al. Effects of age, gender, education and race on two tests of language ability in community-based older adults. Int Psychogeriatr. 2009;21:1051-1062.

39. Persad CC, Abeles N, Zachs RT, Denburg NL. Inhibitory changes after age 60 and their relationship to measures of attention and memory. J Gerontol B Psychol Soc Sci. 2002;57:223-232.

40. Ardila A, Ostrosky-Solis F, Rosselli M, Gomez C. Age-related cog- nitive decline during normal aging: the complex effect of education. Arch Clin Neuropsychol. 2000;15:495-514.

41. Gomez-Perez E, Ostrosky-Solis F. Attention and memory evaluation across the life span: heterogenous effects of age and education. J Clin Exp Neuropsychol. 2007;28:477-494.

42. Kelkar AS, Hough MS, Fang X. Do we think alike? A cross-cultural study of executive functioning. Cult Brain. 2013;1:118-137.

43. Agostini JV, Leo-Summers LS, Inouye SK. Cognitive and other adverse effects of diphenhydramine use in hospitalized older patients. Arch Intern Med. 2001;161:2091-2097.

44. Bossinni L, Coluccia A, Casolaro I, Benbow J, Amodeo G, De Giorgi R, et al. Off-label trazodone prescription: evidence, benefits and risks. Curr Pharm Des. 2015;21:3343-3351.

45. Fritz N, Glogau S, Hoffmann J, Rademacher M, Elger CE, Helmstaeter C. Efficacy and cognitive side effects of tiagabine and topiramate in patients with epilepsy. Epilepsy Beh. 2005;6:373-381.

46. Umegaki H, Kawamura T, Kawano N, Umemura T, Kanai A, Sano T. Factors associated with cognitive decline in elderly diabetics. Dement Geriatr Cogn Dis Extra. 2011;1:1-9.

47. Der G, Deary IJ. Age and sex differences in reaction time in adulthood: results from the United Kingdom health and lifestyle survey. Psychol Aging. 2006;21:62-73.

48. MacDonald SW, Nyberg L, Sandblom J, Fischer H, Backman L. Increased response-time variability is associated with reduced inferior parietal activation during episodic recognition in aging. J Cogn Neurosci. 2008;20:779-786. 